Rev. Int. Contam. Ambie. 33 (1) 75-83, 2017

DOI: 10.20937/RICA.2017.33.01.07

\title{
CALIDAD QUIIMICA DEL AGUA EN UN ÁREA AGRÍCOLA DE MAÍZ FORRAJERO (Zea mays L.) EN LA COMARCA LAGUNERA, MÉXICO
}

\author{
María Elizabeth AZPILCUETA PÉREZ ${ }^{1}$, Aurelio PEDROZA SANDOVAL ${ }^{1 *}$, Ignacio SÁNCHEZ COHEN², \\ María del Rosario SALCEDO JACOBO ${ }^{2}$ y Ricardo TREJO CALZADA ${ }^{1}$
}

${ }^{1}$ Unidad Regional Universitaria de Zonas Áridas, Universidad Autónoma de Chihuahua. Kilómetro 40, Carretera Gómez Palacio, Chihuahua, México, C.P. 35230

${ }^{2}$ Centro Nacional de Investigación Disciplinaria en Relaciones Agua-Suelo-Planta-Atmósfera, Instituto Nacional de Investigaciones Forestales, Agrícolas y Pecuarias. Canal Sacramento, kilómetro 6.5, Zona Industrial, 4ta. etapa. Gómez Palacio, Durango, C.P. 35140

*Autor para correspondencia: aureliops@outlook.com

(Recibido julio 2015; aceptado mayo 2016)

Palabras clave: química del agua, cationes, metales pesados, contaminación química

\section{RESUMEN}

La Comarca Lagunera es un área agrícola que hace uso intensivo de los recursos suelo y agua, éste último de alto impacto por la escasez del mismo. El objetivo del presente estudio fue hacer una valoración de la calidad química del agua de riego de pozo profundo en campos productores de maíz forrajero en la Comarca Lagunera, México. Se realizaron cuatro muestreos de agua durante los meses de abril, mayo, junio y julio del 2014. Se midió conductividad eléctrica (CE) en $\mu \mathrm{S} / \mathrm{cm}$, dureza del agua en grados franceses $\left({ }^{\circ} \mathrm{f}\right)$, así como concentraciones de los cationes $\mathrm{Ca}^{+2}, \mathrm{Mg}^{+2}, \mathrm{Na}^{+}$y $\mathrm{K}^{+}$en meq/L y metales pesados $\mathrm{Cd}, \mathrm{Pb}$ y $\mathrm{As}$ en $\mathrm{mg} / \mathrm{L}$. La salinidad del agua en la Comarca Lagunera medida en términos de CE es de alto a muy alto, con valores de 1732 y $3386 \mu \mathrm{S} / \mathrm{cm}$, en tanto que la sodificación expresada en Índice de Absorción de Sodio (IAS) es medio con un valor de $3.66 \mathrm{meq} / \mathrm{L}$, la dureza del agua varió de dura a muy dura con promedio de 50.47 y $114.00^{\circ} \mathrm{f}$, respectivamente. Las mayores concentraciones de elementos químicos presentes en el agua de riego procedente de pozo profundo fueron $\mathrm{Ca}^{+2}, \mathrm{Mg}^{+2}$ y $\mathrm{Pb}$, principalmente el último. Sin embargo, el Cd y As también rebasaron los límites máximos permisibles por la norma oficial del agua para uso agrícola (SCFI2001 y NOM-117-SSA1-1994), con el consecuente riesgo para la salud y el ambiente.

Key words: chemical of water, cationic elements, heavy metals, chemical contamination

\begin{abstract}
The Comarca Lagunera region from Mexico is an agricultural area which makes intensive use of soil and water resources, this latter of high impact due the it's scarcity. The aim of this study was to assess the chemical quality of irrigation water from deep wells in producing fields of fodder maize in the Laguna region, Mexico. Four samplings were conducted in April, May, June and July 2014. The variables measured were the electrical conductivity (EC) in $\mathrm{mS} / \mathrm{cm}$, water hardness in French degrees $\left({ }^{\circ} \mathrm{f}\right)$, cations $\mathrm{Ca}^{+2}, \mathrm{Mg}^{+2}$, $\mathrm{Na}^{+}$and $\mathrm{K}^{+}$in meq/L, as well as heavy metals $\mathrm{Cd}, \mathrm{Pb}$, and $\mathrm{As}$, in $\mathrm{mg} / \mathrm{L}$. The salinity in
\end{abstract}


the Comarca Lagunera measured in terms of EC was from high to very high with values of 1,732 and 3,386 $\mu \mathrm{S} / \mathrm{cm}$; while the sodification expressed in Sodium Absorption Rate (SAR) is in medium level with $3.66 \mathrm{meq} / \mathrm{L}$ and water hardness ranged from hard to very hard on average 50.47 and $114.00^{\circ} \mathrm{f}$, respectively. The highest concentrations of chemical elements in irrigation water from deep well were $\mathrm{Ca}^{+2}, \mathrm{Mg}^{+2}$ and $\mathrm{Pb}$, mainly the latter. However Cd and As also, were above of the official norm ((NOM-CCA-032-ECOL/1993), with the consequent risk in the health and environment.

\section{INTRODUCCIÓN}

El agua es básica para el mantenimiento de los ecosistemas, los cuales a su vez, son un prerrequisito para la regeneración de este recurso vital en los diferentes procesos físicos y biológicos, tanto en el uso y consumo humano, como en la producción agrícola, pecuaria, forestal e industrial (FOCUS 2006). El acceso a este recurso y su buen manejo, contribuye a un mejor bienestar y a la seguridad alimentaria, mientras que un mal manejo puede generar pobreza y subdesarrollo (UNESCO ONU-Agua 2012).

En México, cerca del $77 \%$ del agua es utilizada en la agricultura, $14 \%$ en abasto público, $4 \%$ en la industria y $5 \%$ en la energía eléctrica (CNA 2013). La actividad agrícola es la de mayor demanda, convirtiéndose en elemento clave en zonas donde este recurso es escaso (PNUMA 2009). Uno de los principales problemas en la Comarca Lagunera de Durango y Coahuila, que es la región de estudio, es la falta de disponibilidad de agua, lo cual se agudiza en los periodos secos, con el consecuente efecto en la recarga del acuífero. De los 653 acuíferos, 101 están sobreexplotados, con una recarga natural de $800 \mathrm{Mm}^{3}$ en el acuífero principal, ante una extracción aproximada de $1252 \mathrm{~mm}^{3}$ y un abatimiento promedio de $1.3 \mathrm{~m}$ por año (CONAGUA 2010). Es común que el agua extraída de pozos a más de $200 \mathrm{~m}$ de profundidad, contengan nutrientes, pero también contaminantes (Sardiñas-Peña et al. 2006). Lo anterior es agravado en lugares donde se ha utilizado agua residual para el riego agrícola, reportándose una tendencia creciente en las concentraciones de metales pesados. Lo anterior es un riesgo potencial para la salud, sobre todo por su acumulación en las plantas (Mancilla-Villa et al. 2012). Así, se hace necesario determinar cuantitativamente la calidad del agua de riego extraída del manto acuífero profundo y su posible impacto al ambiente como base para hacer un uso más eficiente del recurso hídrico y mantener una producción agropecuaria competitiva (Pedroza et al. 2014). Un ejemplo de lo anterior es el caso de la ganadería estabulada en la región, que es de alta demanda de forraje para alimentar a las cerca de 409895 cabezas de ganado lechero (CACL-AC 2013). La producción de forraje en esta área, se basa en cultivos como el maíz (Zea mays), sorgo (Sorghum bicolor), alfalfa (Medicago sativa), cereales de invierno, como trigo (Tritichum vulgare) y avena (Avena sativa), entre otros (MontemayorTrejo et al. 2012).

La contaminación química a partir de agua contaminada, es a través de la cadena alimenticia, mediante la absorción de elementos químicos que realizan las plantas, como pueden ser metales pesados $(\mathrm{Cd}, \mathrm{Pb}$ y As), los cuales están disponibles tanto para los herbívoros como para el ganado y el propio ser humano (Prieto-Méndez et al. 2009). Con base en ello, el objetivo del presente trabajo fue hacer un estudio sobre la calidad química del agua de riego de pozo profundo en campos productores de maíz forrajero en la Comarca Lagunera, México

\section{MATERIALES Y MÉTODOS}

Ubicación geográfica. El estudio se llevó cabo en los municipios de San Pedro, en el estado de Coahuila y Gómez Palacio, Lerdo, Bermejillo y Tlahualilo en el estado de Durango, México. La región se localiza a $24^{\circ} 22^{\prime}$ de latitud norte y $102^{\circ} 22^{\prime}$ de longitud oeste a una altura de 1120 msnm (SEMARNAT 2010-2015).

Base histórica de datos. De acuerdo con la base histórica de datos de la calidad del agua (periodo 1994-2003) proporcionada por la Comisión Nacional del Agua (CNA 2013) y el uso de mapas digitalizados y herramientas de Sistema de Información Geográfica (SIG), se delimitó el área de estudio. Para ello se usaron los datos de cada sitio o vértice de la ubicación de los pozos profundos de extracción del agua de riego del acuífero principal de la región. Los pozos de riego seleccionados para realizar el monitoreo de la calidad del agua, fueron los identificados por la CNA con los números: 2576, 2612, 1103, 1125, 1200, 3387, $950 \mathrm{y}$ 760 (Cuadro I, Fig. 1). El muestreo de agua se efectuó durante los meses de abril, mayo, junio y julio de 2014, 
CUADRO I. UBICACIÓN DE LOS POZOS SELECCIONADOS EN EL ÁREA DE ESTUDIO DE LA COMARCA LAGUNERA, MÉXICO

\begin{tabular}{lllll}
\hline Pozo & Localidad & Ubicación & Longitud & Latitud \\
\hline 2612 & León Guzmán & Lerdo, Dgo. & 103.6729 & 25.53744 \\
2576 & La Loma & Lerdo, Dgo. & 103.67675 & 25.434972 \\
3887 & Venecia & Tlahualilo, Dgo. & 103.341861 & 25.78361 \\
1103 & La Gallega & Gómez Palacio, Dgo. & 103.585916 & 25.802416 \\
1125 & Bermejillo & Bermejillo, Dgo. & 103.341972 & 25.785583 \\
1200 & La Jarita & Tlahualilo, Dgo. & 103.597111 & 25.8965 \\
950 & La Rosita & San Pedro, Coah. & 103.10238 & 25.774611 \\
760 & La Purísima & Matamoros, Coah. & 103.31933 & 25.67038 \\
\hline
\end{tabular}

Dgo. $=$ Durango; Coah.$=$ Coahuila

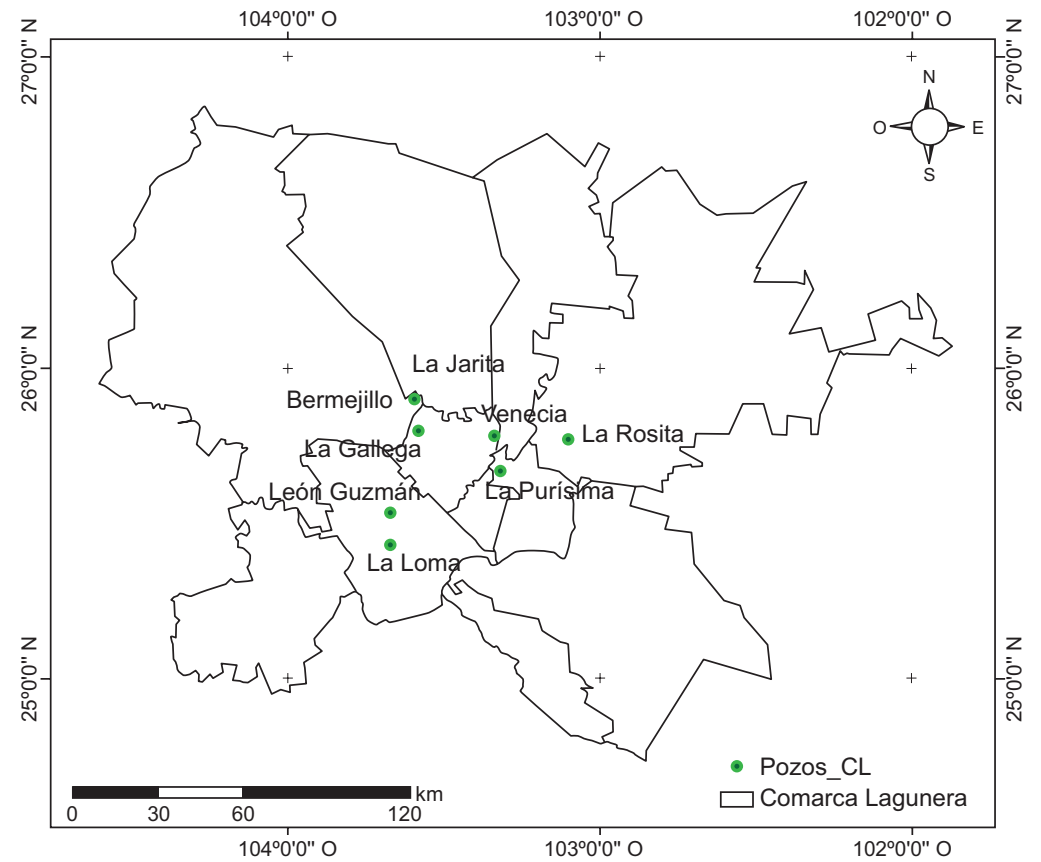

Fig. 1. Localización geográfica de los ocho pozos monitoreados para determinar la calidad química del agua para uso agrícola en la Comarca Lagunera, Durango, México.

periodo que abarca el ciclo primavera-verano de los principales cultivos forrajeros como el maíz y el sorgo.

Monitoreo y análisis químico del agua. En cada fecha de muestreo se tomaron dos muestras de agua. La primera fue utilizada para la determinación de calcio $\left(\mathrm{Ca}^{+2}\right)$, magnesio $\left(\mathrm{Mg}^{+2}\right)$, sodio $\left(\mathrm{Na}^{+}\right)$y potasio $\left(\mathrm{K}^{+}\right)$, mientras que la segunda se empleó para determinar los metales pesados cadmio $(\mathrm{Cd})$, plomo $(\mathrm{Pb})$ y arsénico (As). El análisis químico del agua se realizó con base en las Normas Mexicanas NMX-AA-051SCFI-2001 (SE 2001) y NOM-117-SSA1-1994 (SS 1994). La primera para le recolección de las muestras en los diferentes sitios donde se ubican los pozos y la segunda para en análisis químico, ya que que esta última establece las concentraciones máximas permisibles de $\mathrm{Cd}, \mathrm{Pb}$ y As en el agua. La determinación de los cationes y metales, se realizó en los laboratorios del Instituto Nacional de Investigaciones Forestales Agrícolas y Pecuarias (INIFAP) en Gómez Palacio, Durango. Se empleó un espectrofotómetro de absorción atómica FAAS Perkin Elmer AAnalyst 700, mediante el método de horno de grafito (SE 2001). Las determinaciones de $\mathrm{Ca}^{+2}, \mathrm{Mg}^{+2}, \mathrm{Na}^{+}$y K${ }^{+}$fueron expresadas en meq/L y las de Cd, $\mathrm{Pb}$ y As en mg/L. Las curvas de calibración fueron obtenidas utilizando estándares certificados de $\mathrm{Cd}, \mathrm{Pb}$ y As preparados a partir de una solución concentrada de $1000 \mathrm{mg}$ de 
metal/L, marca Perkin Elmer Pure. Adicionalmente, se midió la conductividad eléctrica (CE) del agua en $\mathrm{mS} / \mathrm{cm}$ con un conductímetro Marca Conductronic Modelo PC45 y se calculó tanto el Índice de Absorción de Sodio (IAS) con la fórmula:

$I A S=N a / \sqrt{\frac{\mathrm{Ca}+\mathrm{Mg}}{2}}$

como la dureza del agua en grados franceses $\left({ }^{\circ} \mathrm{f}\right)$ mediante la ecuación:

Dureza $=\frac{[(C a)(2.5)+(M g)(4.12)]}{10}$

Análisis estadístico de datos. Se realizó un análisis de varianza y otro de rango múltiple de medias Tukey, así como, tanto una regresión lineal como logística, mediante el paquete estadístico SAS V. 9.0. Para el análisis de varianza, se tuvo como fuentes de variación a las localidades donde se ubicaron los pozos y a las fechas de muestreo.

\section{RESULTADOS Y DISCUSIÓN}

Salinización. De acuerdo con el rango de clasificación de calidad del agua para uso agrícola citado por Ayers y Westcot (1987): bajo (0-750), medio (750$1500)$, alto (1500-3000), muy alto $(>3000 \mu \mathrm{s} / \mathrm{cm})$, de los ocho pozos para riego monitoreados durante 2014, el pozo 950 registró una salinización muy alta, con una concentración de $3792.50 \mu \mathrm{S} / \mathrm{cm}$. En tanto que los pozos 2612, 2576, 3887, 1200, 1125, 1103 y 760 mostraron un nivel medio-alto de salinización, con un rango de variación de 1201.25 a $2147.75 \mu \mathrm{S} / \mathrm{cm}$ (Fig. 2). De acuerdo con Mancilla-Villa et al. (2012), los altos valores de CE pueden estar asociados a que el manto acuífero es de influencia de agua subterránea salina. Estos resultados indican que por la condición salobre del agua, al menos el caso del pozo 950, superó el límite de los $3 \mu \mathrm{S} / \mathrm{cm}$ citado por Ayers y Westcot (1987). Sin embargo, la condición sostenida de una salinidad media-alta en la mayoría de los pozos, hace ver que existe un riesgo potencial de daño por ensalitramiento a los suelos agrícolas, con el consecuente impacto en los cultivos y a las líneas regantes por taponeo de sarro salino en el sistema de riego presurizado (AGROCUNSULTING 2015), que es el más común en el área de estudio (Montemayor-Trejo 2012).

Índice de adsorción de sodio (IAS). Siete de los ocho pozos monitoreados, presentaron nivel medio de sodificación de acuerdo con los valores del IAS (con temperatura promedio del agua de los pozos de $28^{\circ} \mathrm{C}$ ), en un rango de variación de 2.34 y $4.46 \mathrm{meq} / \mathrm{L}$, en los pozos 3887 de Venecia y 1103 de la Gallega, respectivamente. Sólo el pozo 760 de la Purísima presentó nivel de sodificación bajo con un valor de $1.59 \mathrm{meq} / \mathrm{L}$ (Fig. 3). El riesgo de sodificación predominante en los pozos es de nivel medio, en función de los contenidos medios de iones de sodio en el agua de riego, lo cual puede afectar la permeabilidad del suelo y ocasionar problemas de infiltración con el consecuente impacto en la agricultura. Esto se debe a que el $\mathrm{Na}^{+}$presente en el suelo, desplaza los iones $\mathrm{Ca}^{+2} \mathrm{y} \mathrm{Mg}^{+2}$ que forman una estructura apropiada para

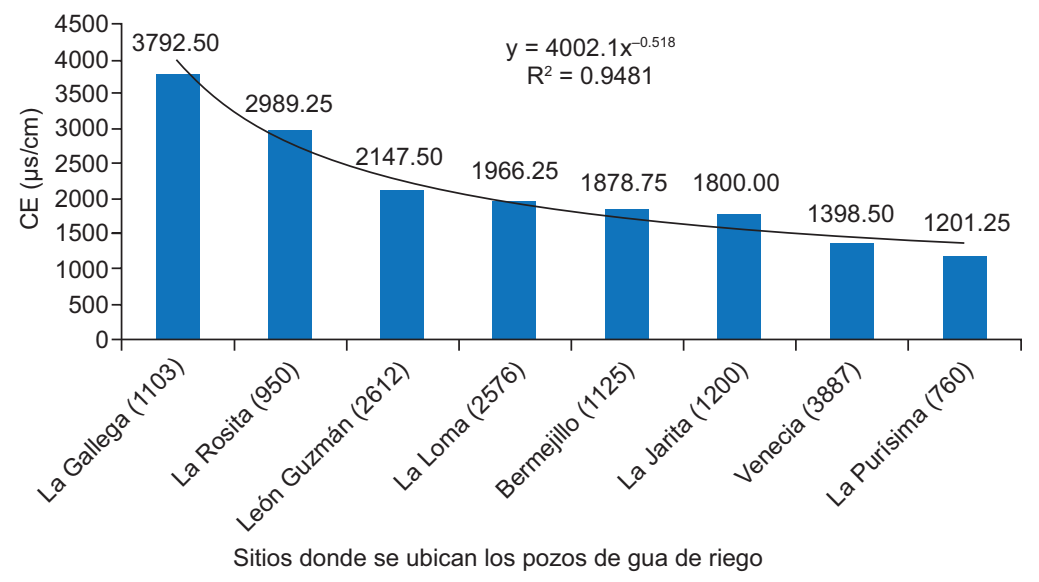

Fig. 2. Nivel de salinizacion en agua de pozo profundo para uso agrícola en diferentes localidades del área de estudio. Comarca Lagunera, Mexico, 2014. $\mathrm{CE}=$ Conductividad eléctrica. Las cifras entre paréntesis de cada comunidad, indican el número de pozo profundo identificado por la $\mathrm{Co}$ misión Nacional del Agua. 


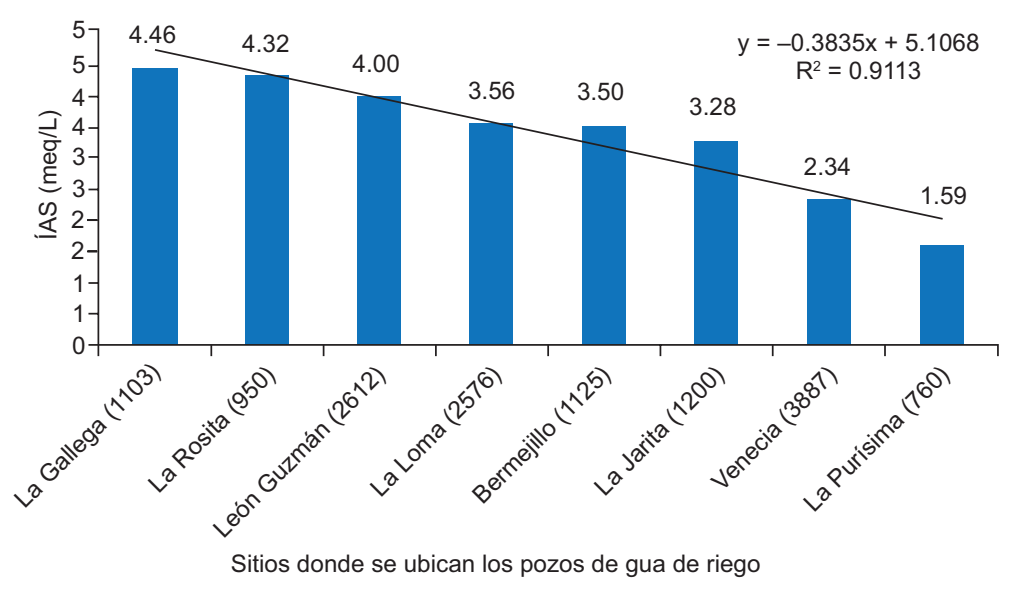

Fig. 3. Indice de adsorción de sodio (IAS) en agua de pozo en diferentes localidades del área de estudio. Comarca Lagunera, México, 2014. Las cifras entre paréntesis de cada comunidad, indican el número de pozo profundo identificado por la Comisión Nacional del Agua.

el cultivo en el suelo $y$, en condiciones secas por abajo del punto de marchitez permanente (PMP), el suelo se vuelve duro y compacto reduciendo la infiltración de agua y aire a través de los poros que lo conforman (Jarsun et al. 2008, AGROCOLSULTING 2015). El riesgo de sodificación en los suelos, sobre todo en la capa superficial, se incrementa conforme se prolonga el periodo de años con riego de agua de pozo profundo con concentraciones medias $(750-1500 \mu \mathrm{S} / \mathrm{cm})$ y altas (1500-3000 $\mu \mathrm{S} / \mathrm{cm})$ de $\mathrm{Na}^{+}$lo que propicia un impacto negativo en los cultivos (Bonet-Pérez et al. 2011).

Dureza del agua. De acuerdo con la clasificación de dureza del agua citada por Canovas (1986), los pozos 1103 y 950 registraron niveles de dureza altos, con valores de 157.93 y $129.32^{\circ}$, , respectivamente. En tanto que el resto de los pozos fueron de niveles medios, con un promedio de $54.3^{\circ} \mathrm{f}$ (Fig. 4). Lo anterior significa que, independientemente de la mayor frecuencia de valores medios en los pozos monitoreados durante los cuatro meses de muestreo, los niveles de $\mathrm{Ca}^{+2}$ en el agua son elevados en la mayor parte del área de estudio, encontrándose concentraciones superiores a $5 \mathrm{meq} / \mathrm{L}$, con el consecuente impacto negativo en el agroecosistema. Lo anterior debido a que el uso de agua de mala calidad ocasiona problemas de salinidad, además disminuye la tasa de infiltración y causa una toxicidad específica sobre los cultivos, entre otros efectos negativos (Moreno y Moral 1996).

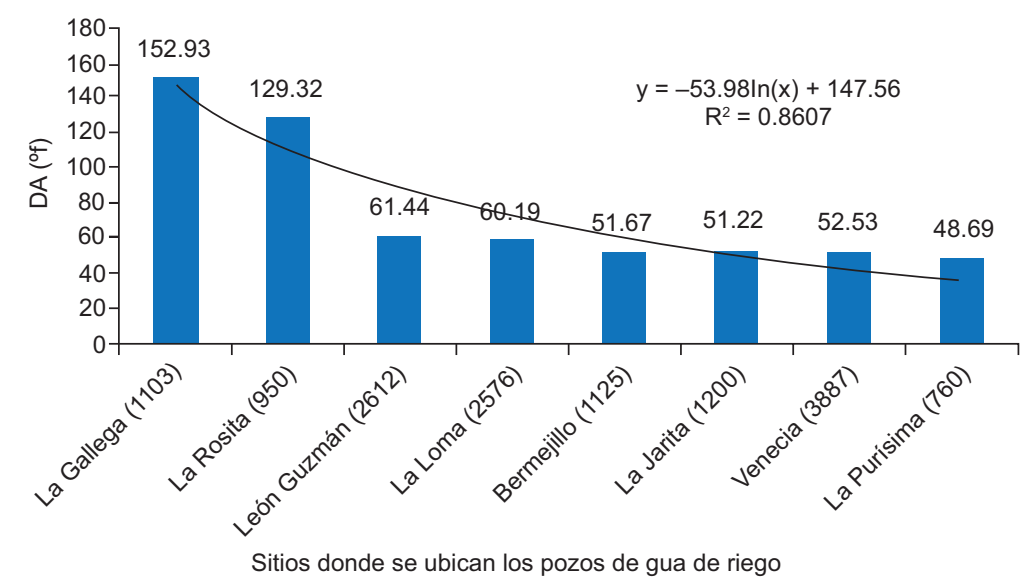

Fig. 4. Dureza del agua (DA) de pozo profundo para uso agrícola en diferentes localidades del área de estudio. Comarca Lagunera, México, 2014. Las cifras entre paréntesis de cada comunidad, indican el número de pozo profundo identificado por la Comisión Nacional del Agua. 


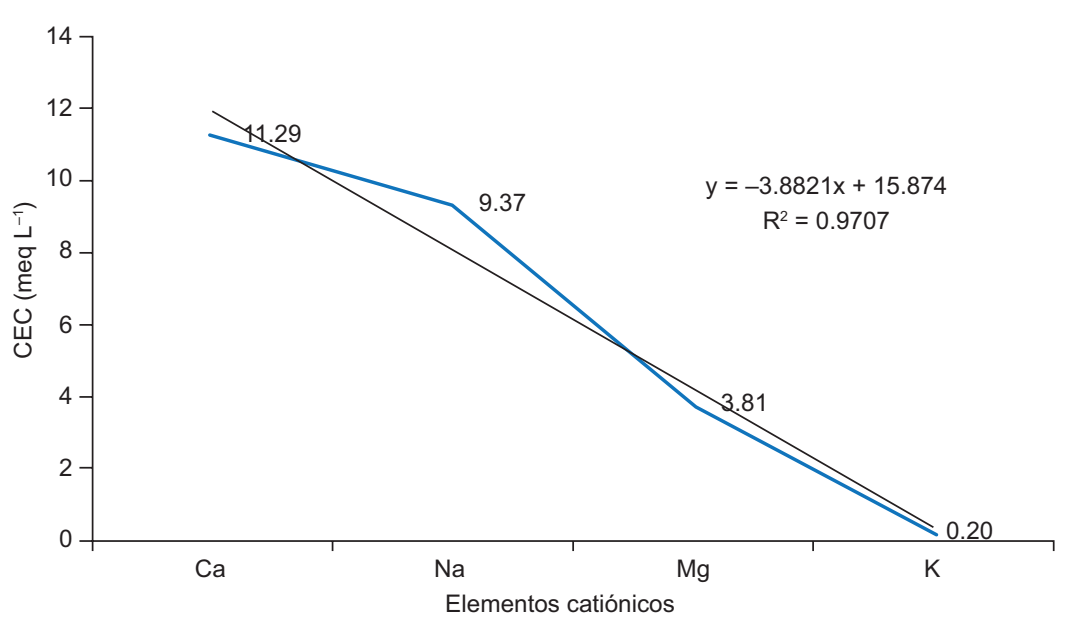

Fig. 5. Concentración de calcio $\left(\mathrm{Ca}^{+2}\right)$, magnesio $\left(\mathrm{Mg}^{+2}\right)$, sodio $\left(\mathrm{Na}^{+}\right)$y potasio $\left(\mathrm{K}^{+}\right)$en agua de pozo para uso agrícola. Comarca Lagunera, México, 2014. $\mathrm{CEC}=$ Concentrado de elementos catiónicos.

Cationes. De los cuatro elementos catiónicos analizados $\left(\mathrm{Ca}^{+2}, \mathrm{Mg}^{+2}, \mathrm{Na}^{+}\right.$y $\left.\mathrm{K}^{+}\right)$en el agua para uso agrícola a nivel regional, el $\mathrm{Ca}^{+2}$ y el $\mathrm{Na}^{+}$se encontraron en mayor concentración, con valores de 11.29 y $9.37 \mathrm{meq} / \mathrm{L}$, respectivamente. En tanto que el $\mathrm{Mg}^{+2}$ y el $\mathrm{K}^{+}$tuvieron concentraciones menores, con valores de 3.81 y 0.203 meq/L, respectivamente (Fig. 5). Los anteriores resultados sobrepasan los límites de la norma establecida por la Organización de las Naciones Unidas para la Alimentación y la Agricultura (Ayers y Westcot 1987), principalmente en referencia al $\mathrm{Ca}^{+2}$ y el $\mathrm{Na}^{+}$, cuyos límites permisibles para el agua de riego (LAR) son de $<5.0 \mathrm{y}<4.0 \mathrm{meq} / \mathrm{L}$ respectivamente. Mientras que el $\mathrm{Mg}^{+2}$ está un poco por encima del valor límite, el cual corresponde a $<3.0 \mathrm{meq} / \mathrm{L}$ y el $\mathrm{K}^{+}$se encuentra dentro del límite
$(<1.0 \mathrm{meq} / \mathrm{L})$. De esta manera, el bicarbonato aportado por el agua de riego bloquea al $\mathrm{Ca}^{+2}$ y al $\mathrm{Mg}^{+2}$ del suelo, aumentando la concentración de $\mathrm{Na}^{+}$, lo que puede originar que el bulbo de humedad (área de la rizósfera con humedad disponible para la planta) evolucione hasta un suelo sódico (Agroconsulting 2015).

La concentración de cationes en el tiempo durante los meses muestreados en el 2014, no varió para el caso del $\mathrm{Ca}^{+2}$ y el $\mathrm{Mg}^{+2}(\mathrm{P} \geq 0.05)$. En cambio el $\mathrm{Na}^{+}$ y el $\mathrm{K}^{+}$presentaron concentraciones significativamente mayores en los meses de abril, junio y julio, con valores de $9.42,11.49$ y $9.63 \mathrm{meq} / \mathrm{L}$, respectivamente $(\mathrm{P} \leq 0.05) . \mathrm{El} \mathrm{K}^{+}$fue significativamente más alto en los meses de abril con $0.257 \mathrm{meq} / \mathrm{L}$ y julio con 0.226 meq/L (Fig. 6). Lo anterior significa que el $\mathrm{Ca}^{+2}$ y el $\mathrm{Mg}^{+2}$ son más estables, ya que no variaron $(\mathrm{P} \geq 0.05)$

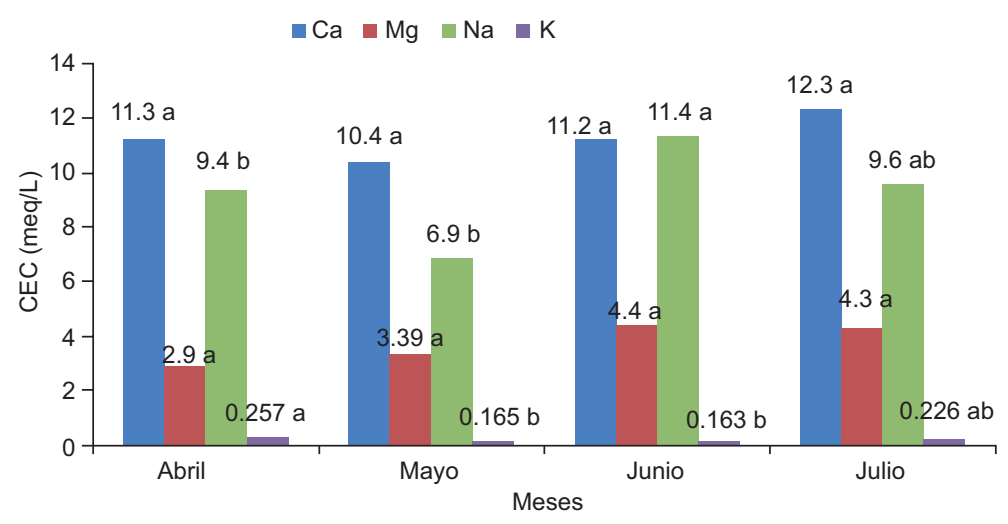

Fig. 6. Variación temporal de la concentración (meq/L) de cationes en agua de pozo para uso agrícola. Comarca Lagunera, México, 2014. Prueba de Tukey $(\mathrm{P} \leq 0.05)$. Cifras con las mismas letras dentro de una columna del mismo color, son estadísticamente iguales. $\mathrm{CEC}=$ Concentración de elementos catiónicos. 
CUADRO II. VARIACIÓN ESPACIAL DE LA CONCENTRACIÓN MEDIA (meq/L) DE CATIONES EN AGUA DE POZO PARA USO AGRÍCOLA. COMARCA LAGUNERA MÉXICO, 2014

\begin{tabular}{lcccc}
\hline Localidad & $\mathrm{Ca}^{+2}$ & $\mathrm{Mg}^{+2}$ & $\mathrm{Na}^{+}$ & $\mathrm{K}^{+}$ \\
\hline Bermejillo & $8.53 \mathrm{~b}$ & $3.50 \mathrm{bc}$ & $8.87 \mathrm{bc}$ & $0.23 \mathrm{a}$ \\
& $( \pm 0.880)$ & $( \pm 0.636)$ & $( \pm 0.346)$ & $( \pm 0.037)$ \\
La Purísima & $8.56 \mathrm{~b}$ & $0.98 \mathrm{c}$ & $3.49 \mathrm{~d}$ & $0.17 \mathrm{a}$ \\
& $( \pm 0.194)$ & $( \pm 0.042)$ & $( \pm 0.767)$ & $( \pm 0.042)$ \\
La Gallega & $20.89 \mathrm{a}$ & $9.09 \mathrm{a}$ & $17.27 \mathrm{a}$ & $0.25 \mathrm{a}$ \\
& $( \pm 1.026)$ & $( \pm 1.088)$ & $( \pm 1.696)$ & $( \pm 0.023)$ \\
La Jarita & $7.98 \mathrm{~b}$ & $3.25 \mathrm{bc}$ & $8.51 \mathrm{bcd}$ & $0.21 \mathrm{a}$ \\
& $( \pm 0.275)$ & $( \pm 0.423)$ & $( \pm 0.573)$ & $( \pm 0.027)$ \\
La Loma & $7.89 \mathrm{~b}$ & $4.83 \mathrm{~b}$ & $10.89 \mathrm{~b}$ & $0.22 \mathrm{a}$ \\
& $( \pm 0.213)$ & $( \pm 0.445)$ & $( \pm 0.414)$ & $( \pm 0.027)$ \\
León Guzmán & $6.83 \mathrm{~b}$ & $3.21 \mathrm{bc}$ & $8.96 \mathrm{bc}$ & $0.18 \mathrm{a}$ \\
& $( \pm 1.268)$ & $( \pm 1.095)$ & $( \pm 3.093)$ & $( \pm 0.018)$ \\
La Rosita & $20.60 \mathrm{a}$ & $4.57 \mathrm{~b}$ & $11.70 \mathrm{~b}$ & $0.21 \mathrm{a}$ \\
& $( \pm 1.210)$ & $( \pm 0.413)$ & $( \pm 1.420)$ & $( \pm 0.041)$ \\
Venecia & $9.08 \mathrm{~b}$ & $1.04 \mathrm{c}$ & $5.28 \mathrm{~d}$ & $0.18 \mathrm{a}$ \\
& $( \pm 0.443)$ & $( \pm 0.108)$ & $( \pm 0.252)$ & $( \pm 0.029)$ \\
\hline
\end{tabular}

Prueba de Tukey $(\mathrm{P} \leq 0.05)$. Cifras con las mismas letras dentro de una misma columna, son estadísticamente iguales. Las cifras entre paréntesis corresponden al Error Estándar

durante el periodo muestreado (abril, mayo, junio y julio), que corresponde al periodo lluvioso, lo cual no parece afectar la tasa de infiltración durante la fase de recarga del acuífero. Por el contrario, el $\mathrm{Na}^{+}$y el $\mathrm{K}^{+}$ sí fueron afectados en sus concentraciones, las cuales resultaron significativamente mayores en los meses de junio y julio para el $\mathrm{Ca}^{+2}$ y en abril y julio para el $\mathrm{K}^{+}$.

En relación con la variación por localidad, en la Gallega el $\mathrm{Ca}^{+2}$, el $\mathrm{Mg}^{+2}$ y el $\mathrm{Na}^{+}$fueron significativamente mayores en sus concentraciones con $20.89,9.09$ y $17.27 \mathrm{meq} / \mathrm{L}$, respectivamente. La localidad la Rosita también lo fue en $\mathrm{Ca}^{+2}$. El resto de las regiones mostraron valores de concentraciones medios y bajos para estos elementos, respecto a los dos sitios antes citados. Estos resultados pueden estar asociados con la variación de las propiedades químicas y físicas del suelo en la región de estudio. Por otro lado, el $\mathrm{K}^{+}$no varió en toda la región (Cuadro II).

Metales pesados. $\mathrm{El} \mathrm{Pb}$ mostró la mayor concentración en el agua, con un valor de $0.302 \mathrm{mg} / \mathrm{L}$, en tanto que el $\mathrm{Cd}$ y el As estuvieron en concentraciones menores de 0.039 y $0.036 \mathrm{mg} / \mathrm{L}$, respectivamente (Fig. 7). Las concentraciones antes citadas están muy por encima de límite máximo permisible por la NOM127-SSA1-1994 (SS 1994), que establece valores máximos de $0.005,0.025$ y $0.025 \mathrm{mg} / \mathrm{L}$ para $\mathrm{Cd}, \mathrm{Pb}$ y As, respectivamente. Destaca el $\mathrm{Pb}$, con un valor de $1208 \%$ superior al límite permitido, en tanto que el As y el Cd están en un 144 y $780 \%$ por encima de la norma, respectivamente. Gómez et al. (2004), reportaron altas concentraciones de $\mathrm{Pb}$ asociado con lugares de actividad minera, lo cual es el caso para esta área de estudio, que se reconoce como una región minera.

No hubo variación durante los meses de estudio en la concentración de As, pero sí para el $\mathrm{Cd}$ y el $\mathrm{Pb}$, los cuales fueron significativamente mayores en los meses de junio $(0.044 \mathrm{mg} / \mathrm{L})$ y julio $(0.339 \mathrm{mg} / \mathrm{L})$, respectivamente (Fig. 8). Lo anterior posiblemente también a la fuente de disposición superficial en el suelo del $\mathrm{Cd}$ y el $\mathrm{Pb}$. En tanto que el As es más dependiente de su disponibilidad en el subsuelo.

En cuanto a la variación por localidad, las concentraciones de $\mathrm{Cd}, \mathrm{Pb}$ y As, no variaron significativamente $(P \geq 0.05)$ entre localidades. Sin embargo,

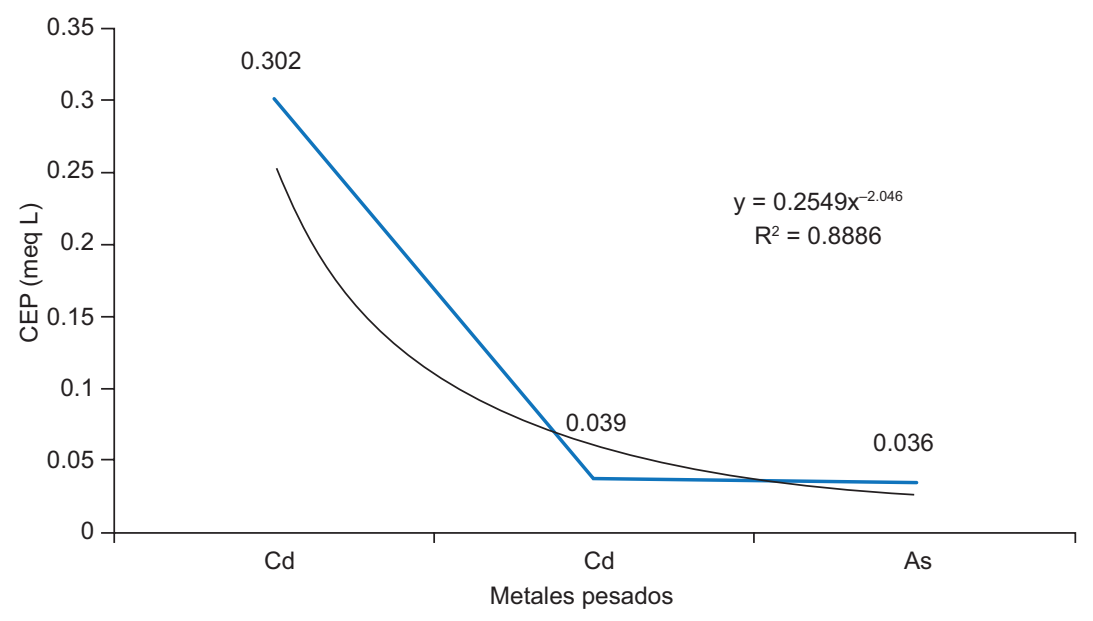

Fig. 7. Concentración de cadmio $(\mathrm{Cd})$, plomo $(\mathrm{Pb})$ y arsénico $(\mathrm{As})$ en agua de pozo profundo para uso agrícola. Comarca Lagunera, México, 2014. CEP = Concentración de elementos pesados. 


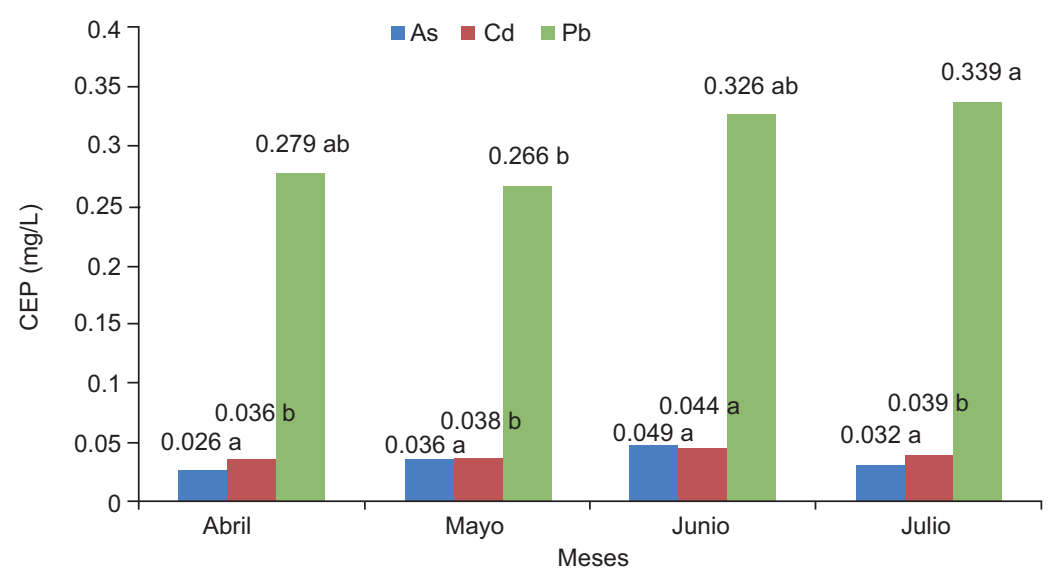

Fig. 8. Variación temporal de metales pesados en agua de pozo para uso agrícola. Comarca Lagunera, México, 2014. Prueba de Tukey $(\mathrm{P} \leq 0.05)$. Cifras con las mismas letras entre columnas del mismo color, son estadísticamente iguales. $\mathrm{CEP}=$ Concentración de elementos pesados.

su concentración es alta en todos los sitios monitoreados, de acuerdo con los límites establecido por la norma NOM-127-SSA1-1994 (SS 1994), en los que destacan el $\mathrm{Pb}$ y el $\mathrm{Cd}$ (Cuadro III). Lo anterior coincide con el análisis de estos metales en el tiempo, lo cual indica que, independientemente de la época del año durante la primavera-verano las concentraciones de los metales es alta en toda la región de estudio. Lo que lleva al consecuente riesgo ambiental y a la

CUADRO III. VARIACIÓN ESPACIAL DE LA CONCENTRACIÓN MEDIA (mg/L) DE METALES PESADOS EN AGUADE POZO PROFUNDO PARA USO AGRÍCOLA. COMARCA LAGUNERA MÉXICO, 2014

\begin{tabular}{lccc}
\hline Localidad & $\mathrm{As}$ & $\mathrm{Cd}$ & $\mathrm{Pb}$ \\
\hline Bermejillo & $0.052 \mathrm{a}$ & $0.038 \mathrm{a}$ & $0.329 \mathrm{a}$ \\
& $( \pm 0.006)$ & $( \pm 0.001)$ & $( \pm 0.035)$ \\
La Purísima & $0.010 \mathrm{a}$ & $0.039 \mathrm{a}$ & $0.323 \mathrm{a}$ \\
& $( \pm 0.0 .001)$ & $( \pm 0.0007)$ & $( \pm 0.042)$ \\
La Gallega & $0.032 \mathrm{a}$ & $0.041 \mathrm{a}$ & $0.320 \mathrm{a}$ \\
& $( \pm 0.004)$ & $( \pm 0.001)$ & $( \pm 0.028)$ \\
La Jarita & $0.032 \mathrm{a}$ & $0.038 \mathrm{a}$ & $0.290 \mathrm{a}$ \\
& $( \pm 0.004)$ & $( \pm 0.002)$ & $( \pm 0.016)$ \\
La Loma & $0.038 \mathrm{a}$ & $0.039 \mathrm{a}$ & $0.302 \mathrm{a}$ \\
& $( \pm 0.002)$ & $( \pm 0.001)$ & $( \pm 0.026)$ \\
León Guzmán & $0.066 \mathrm{a}$ & $0.040 \mathrm{a}$ & $0.287 \mathrm{a}$ \\
& $( \pm 0.035)$ & $( \pm 0.002)$ & $( \pm 0.031)$ \\
La Rosita & $0.050 \mathrm{a}$ & $0.042 \mathrm{a}$ & $0.276 \mathrm{a}$ \\
& $( \pm 0.002)$ & $( \pm 0.003)$ & $( \pm 0.013)$ \\
Venecia & $0.018 \mathrm{a}$ & $0.038 \mathrm{a}$ & $0.292 \mathrm{a}$ \\
& $( \pm 0.006)$ & $( \pm 0.001)$ & $( \pm 0.016)$ \\
\hline
\end{tabular}

Prueba de Tukey $(\mathrm{P} \leq 0.05)$. Cifras con las mismas letras dentro de una misma columna son estadísticamente iguales. Las cifras entre paréntesis corresponden al error estándar salud, éste último por tratarse de un región donde es predominante la producción de cultivos forrajeros para el ganado lechero (CACL-AC 2013).

\section{CONCLUSIONES}

Siete de los ocho pozos monitoreados mostraron un nivel medio-alto de salinización, con un rango de variación de 1201.25 a $2147.75 \mu \mathrm{S} / \mathrm{cm}$. En tanto que sólo un sitio (950) presentó un nivel alto de salinidad, superior a $3000 \mu \mathrm{S} / \mathrm{cm}$. El Ca ${ }^{+2} \mathrm{y} \mathrm{Na}^{+}$ se encontraron en mayor concentración, (11.29 y $9.37 \mathrm{meq} / \mathrm{L}$, respectivamente), en relación con el $\mathrm{Mg}^{+2}$ y el $\mathrm{K}^{+}$, los cuales registraron las menores concentraciones. El $\mathrm{Pb}$ registró la mayor concentración en el agua con un valor de $0.302 \mathrm{mg} / \mathrm{L}$. El Cd $\mathrm{y}$ el As estuvieron en concentraciones menores, con valores de 0.039 y $0.036 \mathrm{mg} / \mathrm{L}$, respectivamente. Sin embargo, los tres en concentraciones muy por encima de límite máximo permisible. Hay una variación en el tiempo y entre localidades en las concentraciones de cationes, principalmente del $\mathrm{Na}^{+}$y del $\mathrm{K}^{+}$, así como de los metales pesados, en especial del $\mathrm{Cd}$ y $\mathrm{Pb}$, lo cual debe ser considerado al implementar posibles medidas de mitigación.

\section{REFERENCIAS}

Agroconsulting (2015). Impacto del agua de riego en el suelo de olivar de regadío [en línea] http://oliviculturadeprecision.com/impacto-del-agua-de-riego-en-elsuelo-de-olivar-de-regadio/ 17/07/2015 
Ayers R. S. y Westcot D. W. (1987). La calidad del agua y su uso en la agricultura. Estudio FAO. Riego y Drenaje 29. Food and Agriculture Organization. Roma, Italia, $223 \mathrm{pp}$.

Bonet-Pérez C., Ricardo C. y Martha P. (2011). Calidad del agua de riego y su posible efecto en los rendimientos agrícolas en la Empresa de Cultivos Varios Sierra de Cubitas. Rev. Cie. Téc. Agr. 20, 19-23.

CNA (2013). Estadísticas del agua en México. Edición 2013. Secretaría de Medio Ambiente y Recursos Naturales. Comisión Nacional del Agua. Tlalpan, Ciudad de México, México, 176 pp.

CONAGUA (2010). Atlas digital del agua México 2010. Contraste regional entre el desarrollo y la disponibilidad del agua, 2008 [en línea] http://www.conagua. gob.mx/atlas/ 15/08/2014

CACL-AC (2013). La importancia del sector agropecuario en la Comarca Lagunera. Consejo Agropecuario de la Comarca Lagunera, A.C. Torreón, México. [en línea]. http://www.avicultura.com.mx/ganaderia/home/impresion.asp?cve_art=1124 20/07/2014

FOCUS (2006). A gua para la alimentación - una cuestión de supervivencia. InfoResources. $\mathrm{N}^{\mathrm{o}} 3 / 06$. [en línea]. http://www.inforesources.ch/pdf/focus06_3_s.pdf 17/05/2014

Gómez A. A., Villalba A. A., Acosta R. G., Castañeda O. M. y Camp D. (2004). Metales pesados en el agua superficial del Río San Pedro durante 1997 y 1999. Rev. Int. Contam. Ambie. 20, 1-8.

Jarsun R., Bustos V. y Carnero M. (2008). Manual de uso e interpretación de aguas. Laboratorio de Suelos, Agua y efluentes. Secretaría del Medio Ambiente. Córdoba, España, $50 \mathrm{pp}$.

Mancilla-Villa M. V., Ortega-Escobar H. M., RamírezAyala R., Uscanga-Mortera E., Ramos-Bello R. y Reyes-Ortigoza A. L. (2012). Metales pesados totales y arsénico en el agua para riego de Puebla y Veracruz, México. Rev. Int. Contam. Ambie. 28, 39-48.

Montemayor-Trejo J. A., Lara-Mireles J. L., Woo-Reza J. L., Munguía-López J., Rivera-González M. y TruciosCaciano R. (2012). Producción de maíz forrajero (Zea mays L.) en tres sistemas de irrigación en la Comarca Lagunera de Coahuila y Durango, México. Agrociencia 46, 267-278.

Moreno J. M. D. y Moral M. (1996). Análisis y calidad del agua para el riego: Editorial Servicios de Publicaciones Universidad Politécnica de Valencia, España, 70 pp.
SE (2001). Norma Oficial Mexicana NMX-AA-051-SCFI-2001. Análisis de agua - determinación de metales por absorción atómica en aguas naturales, potables, residuales y residuales tratadas - método de prueba. Secretaría de Educación. Diario Oficial de la Federación. 25 de marzo de 1980.

SS (1994). Norma Oficial Mexicana NOM-117SSA1-1994. Bienes y servicios. Método de prueba para la determinación de cadmio, arsénico, plomo, estaño, cobre, fierro, zinc y mercurio en alimentos, agua potable y agua purificada por espectrometría de absorción atómica. Secretaría de Salud. Diario Oficial de la Federación. 29 de junio de 1995.

Pedroza-Sandoval A., Flores-Ríos J. L., Torres-Moreno M., Cantú-Brito, J. E., Piceno-Sagarnaga C. y YáñezChávez L. G. (2014). Eficiencia del agua de riego en la producción de maíz forrajero (Zea mays L.) y alfalfa (Medicago sativa): Impacto social y económico. Revista Terra Latinoamericana 32, 231-239.

PNUMA (2009). The environmental food crisis: The environment's role in averting future food crises. Programa de las Naciones Unidas para el Medio Ambiente. México [en línea]. www.grida.no/files/publications/ FoodCrisis_lores.pdf 14/7/2014

Prieto-Méndez J., González-Ramírez C. A., RománGutiérrez A. D., Prieto-García F. (2009). Contaminación y fitotoxicidad en plantas por metales pesados provenientes de suelos y agua. Tropical and Subtropical Agroecosystems 10, 29-44

Sardiñas-Peña O., Chiroles-Rubalcaba S., FernándezNovo M., Hernández-Rodríguez Y. y Pérez-Cabrera A. (2006). Evaluación físico-química y microbiológica del agua de la presa El Cacao (Cotorro, Cuba). Higiene y Sanidad Ambiental 202-206

SEMARNAT 2013. Programas para mejorar la calidad del aire en la región de la Comarca Lagunera 2010-2015. Secretaría de Medio Ambiente y Recursos Naturales. México [en línea]. http://apps1.semarnat.gob.mx/dgeia/compendio_2013/dgeiawf.semarnat.gob.mx_8080/ ibi_apps/WFServletale0.html 23/04/2015

UNESCO ONU-Agua (2012). Programa mundial de evaluación de los recursos hídricos (World Water Assessment Programme) [en línea]. www.unesco.org/ water/wwap/index_es.shtml 18/5/2014 